Oncology

\title{
Liposarcoma of the spermatic cord: A case report and review of literature
}

\author{
Amina Mokrani ${ }^{\mathrm{a}}$, Fatma Guermazi ${ }^{\mathrm{a}, *}$, Khedija Meddeb ${ }^{\mathrm{a}}$, Linda Bel Hadj Kacem ${ }^{\mathrm{b}}$, \\ Marouene Chakroun $^{c}$, Yosra Yahyaoui ${ }^{a}$, Feriel Letaief ${ }^{a}$, Azza Gabsi $^{\mathrm{a}}$, Abderrazek Bouzouita ${ }^{\mathrm{c}}$, \\ Mouna Ayadi ${ }^{\mathrm{a}}$, Nesrine Chraiet ${ }^{\mathrm{a}}$, Henda Raies ${ }^{\mathrm{a}}$, Soumaya Rammeh ${ }^{\mathrm{b}}$, Mohamed Chebil ${ }^{\mathrm{c}}$, \\ Amel Mezlini ${ }^{\mathrm{a}}$
}

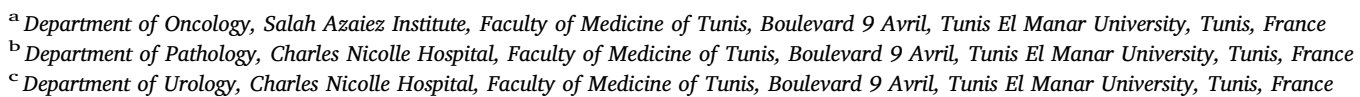

\section{Introduction}

Tumors of the spermatic cord are mostly benign (70-80\%). Among malignant tumors of the spermatic cord, sarcomas are the most current. The most common and aggressive type of sarcoma in children is rhabdomyosarcoma, whereas the most prevalent one in adults is liposarcoma. Liposarcoma of spermatic cord (LSC) is extremely rare. It accounts for $37 \%$ of all spermatic cord tumors. To our knowledge only about 327 cases have been described in the literature including our case. We, therefore, are reporting our experience with a case report of a LSC. $^{1}$

Case presentation

A 64-year-old man noticed a swelling with a painless mass in the right scrotum. He visited the urological surgery department of Charles Nicolle Hospital (HCN) on February 2014. Pre-operative imaging didn't reveal any lymph node or distant metastasis. A right orchiectomy was performed. The orchiepididimectomy piece measured $9 \times 7 \times 6 \mathrm{~cm}$. There was a yellowish multi-lobulated para-testicular mass of $7 \times 6 \mathrm{~cm}$ involving the spermatic cord. Histological findings showed a malignant mesenchymal proliferation. It included well-differentiated areas of mature adipocyte proliferation of varying size and shape "lipoma-like", mixed with broad bands of locally myxoid fibrous tissue with starshaped fusiform cells. They were equipped with hyperchromatic nuclei with the presence of mitotic figures. The area was myxoid containing fibro-hyaline rearrangements without necrosis. There was no invasion of the testicular tissue or the epididymis. All the margins seemed to be negative. The pathological diagnosis was therefore a LSC associating a dedifferentiated component arising from an area of well differentiated contingent (FNCLCC) (Figs. 1 and 2). The patient was lost to follow-up during 2 years.

He came two years later with a recurrence of his right scrotal mass. The CT-scan showed a necrotic tissue mass of metastatic appearance, centered on the right ischio-pubic branch with rupture of the cortex that was the consequence of the local extension of the tumor. The mass measured $42 \times 37 \times 30 \mathrm{~mm}$ and invaded the ipsilateral internal obturator muscle. The patient underwent a resection of the scrotal mass on March 2016. Pathological review demonstrated a poorly differentiated liposarcoma arising from an area of well differentiated grade 2 liposarcoma (Fig. 3). The patient was referred to Salah Azaiez Institute (ISA) for chemo and radiation therapy. The case was discussed at a multidisciplinary team meeting. Radiotherapy has been indicated and is currently underway. The indication for chemotherapy has not been retained.

\section{Discussion}

Liposarcoma is the most common subtype of sarcoma among adult (46.4\%). Some environmental predispositions have been suggested in a minority of patients such as external radiotherapy, pesticides, arsenic, etc. or genetic predispositions. Para testicular liposarcoma occurs typically in adults between 50 and 60 years. It is localized more frequently on the right side.

LSC originates mainly from derivatives of embryonic mesoderm, but may also arise from the testicular tunica or epididymis. Some authors believe that the lesion occurs from the tissues of the spermatic cord; others suggest that it can occur from a malignant degeneration of a preexisting lipoma. ${ }^{2}$

LSC is often present as a large purse. The scrotal swelling can

\footnotetext{
${ }^{*}$ Corresponding author.

E-mail addresses: aidamokrani@yahoo.fr (A. Mokrani), fatmaguermazi21@gmail.com (F. Guermazi), Khedijameddeb@gmail.com (K. Meddeb), molkaabdennebi@yahoo.fr (L.B. Hadj Kacem), chakrounmarouene@yahoo.fr (M. Chakroun), yosyahyaoui@gmail.com (Y. Yahyaoui), feryel.ksontini@gmail.com (F. Letaief), azza_g86@yahoo.fr (A. Gabsi), bouzouitabder@yahoo.fr (A. Bouzouita), mounaayadi28@gmail.com (M. Ayadi), nesrinechraiet@gmail.com (N. Chraiet), henda.rais@yahoo.fr (H. Raies), rammehs@yahoo.fr (S. Rammeh), mohamed.chebil.uro@gmail.com (M. Chebil), amel.mezlini@rns.tn (A. Mezlini).
} 


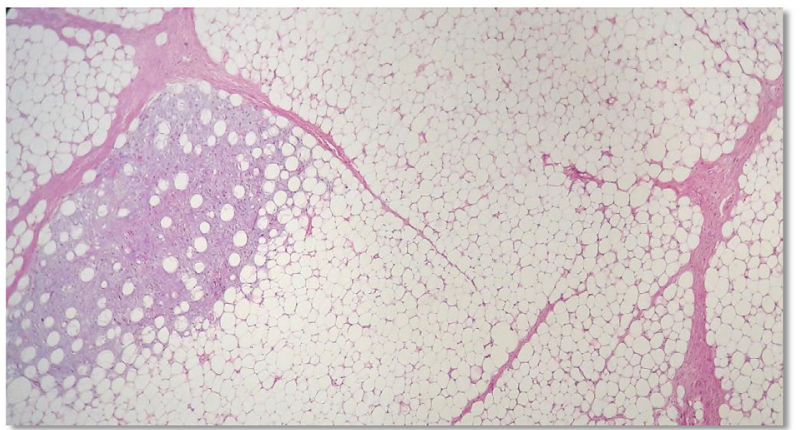

Fig. 1. Initial tumor: Area of well-differentiated liposarcoma (Low magnification).

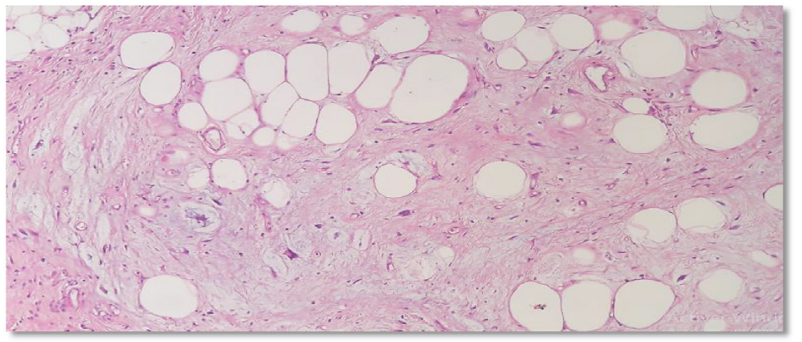

Fig. 2. Initial tumor: Well differentiated liposarcoma (High magnification).

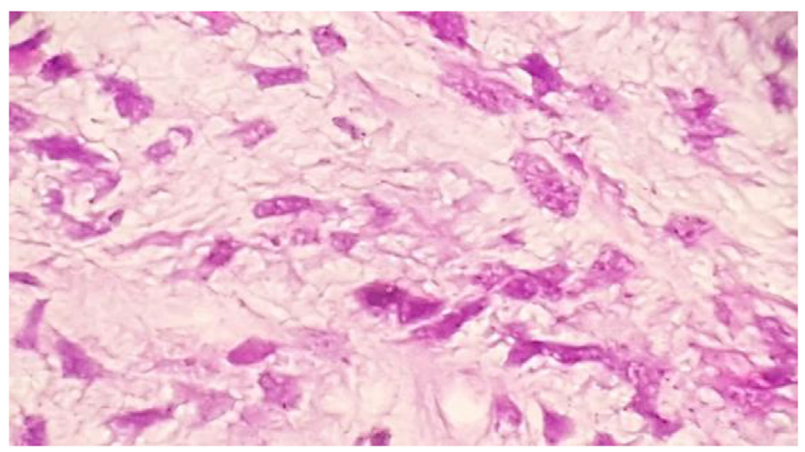

Fig. 3. Recurrence of a liposarcoma in the form of an undifferentiated sarcoma high grade non-lipogenic area (High magnification).

gradually increase very slowly over a period ranging from months to years and then increase rapidly in size. On palpation, the tumor is often painless, firm, non-tender, irregular in consistency and do not transmit light. There are no pathognomonic radiological signs of LSC on imaging. In addition, there is no correlation between mass size and malignancy.

There is no gold standard in the literature toward the management of this entity. Thus, the treatment is based on the guidance of case reports.

Surgery of tumor cord, is recommended if there is suspicion of a malignant tumor or if there is doubt about the benignity of the lesion. Surgical treatment of LSC involves an enlarged orchiectomy. A second resection is recommended when if the margins are positive.
Regional lymph node dissection is not of value because the dissemination of LCS is essentially haematogenous whereas lymphatic pattern of metastases seems to be infrequent. However, some authors believe that lymphatic dissection reduces the risk of loco regional recurrence. Banowsky and Shultz studied the benefit of lymphatic dissection by performing retroperitoneal lymph node dissections in 29 patients. Lymph nodes were positive in 17 patients but had no impact on survival. ${ }^{3}$

Local recurrence rate after conservative surgery alone reported in literature is up to $30 \%$.

Even if LSC is the most radiosensitive sarcoma, the role of adjuvant radiotherapy (RT) remains unclear. There are two prospective randomized trials that demonstrated the role of the addition of radiation to surgery in soft tissue sarcoma. They showed that postoperative external-beam radiotherapy is highly effective in preventing loco-regional (LR) failure. However this advantage did not translate into an overall survival benefit. Yang et al. established the role of postoperative external-beam RT on local recurrence and overall survival (OS). They showed that postoperative RT can significantly decreased the 10-year local recurrence rate in patients with high-grade lesions $(p=0.0028)$ and some selected patient with low grade lesions $(\mathrm{p}=0.015)$. Thus RT is recommended in addition to surgery for high-grade tumors, lymphatic invasion, inadequate margins, or relapses. ${ }^{4}$

Concerning the role of chemotherapy, adjuvant doxorubicin-based chemotherapy for resected localized soft tissue sarcomas in general has been evaluated in a large meta-analysis. It showed an impact in recurrence-free survival and a trend toward an increase in overall survival. However regarding the role of chemotherapy in the treatment of LSC, there is no evidence based data in the literature that proved its benefice and its impact is not documented adequately. ${ }^{1,5}$ Local recurrence is the main problem of para-testicular sarcoma. This recurrence occurs in $30-50 \%$ of cases. It may occur after delays of up to five years. A long follow-up is therefore required in all cases. ${ }^{5}$

\section{Conclusions}

Para testicular liposarcoma is a rare and unusual mesenchymal tumor. The treatment consists of an inguinal orchiectomy. Lymph node dissection is currently not justified. The role Adjuvant RT is controversial but may reduce local recurrence. Chemotherapy didn't prove its benefice until now.

\section{References}

1. Chalouhy C, Ruck JM, Moukarzel M, Jourdi R, Dagher N, Philosophe B. Current management of liposarcoma of the spermatic cord: a case report and review of the literature. Molecular and Clinical Oncology. 2017 Mar 1;6(3):438-440.

2. Hinman F, GIBSON TE. Tumors of the epididymis, spermatic cord and testicular tunics: a review of the literature and report of three new cases. Arch Surg. 1924 Jan 1;8(1):100-137 Johnson DE, Harris JD, Ayala AG. Liposarcoma of spermatic cord. Urology. 1978 Feb 1;11(2):190-2

3. Banowsky LH, Shultz GN. Sarcoma of the spermatic cord and tunics: review of the literature, case report and discussion of the role of retroperitoneal lymph node dissection. J Urol. 1970 May 1;103(5):628-631.

4. Yang DM, Kim HC, Lim JW, et al. Sonographic findings of groin masses. J Ultrasound Med. 2007 May 1;26(5):605-614.

5. Coleman J, Brennan MF, Alektiar K, Russo P. Adult spermatic cord sarcomas: management and results. Ann Surg Oncol. 2003 Jul 1;10(6):669-675. 